\title{
CAMC Focused Section on Tensor Computation
}

\author{
Delin $\mathrm{Chu}^{1} \cdot$ Michael $\mathrm{Ng}^{2} \cdot$ Liqun $\mathrm{Qi}^{3} \cdot$ Qing-Wen Wang ${ }^{4}$
}

Published online: 4 January 2021

(c) Shanghai University 2021

Tensors are multi-arrays with more than two indices. In the last decade or so, many concepts and results in matrix theory—some of which are nontrivial—have been extended to tensors and have a wide range of applications. The present focused section entitled "tensor computation" in Communications on Applied Mathematics and Computation (CAMC) covers tensors in inversion, principal component analysis, spectral hyperspectral theory, decompositions, and rank structure. We do hope this focused section can offer fresh stimuli for the community of tensor computation and theory to promote and develop cutting-edge research in this important field.

Michael $\mathrm{Ng}$

mng@maths.hku.hk

1 National University of Singapore, Singapore 119077, Singapore

2 The University of Hong Kong, Pok Fu Lam 999077, Hong Kong, China

3 Hangzhou Dianzi University, Hangzhou 310018, China

4 Shanghai University, Shanghai 200444, China 